\title{
Stress Fracture of the Femoral Shaft in Paget's Disease of Bone: A Case Report
}

\author{
Sarthak Nepal', Atthakorn Jarusriwanna², Aasis Unnanuntana ${ }^{1}$ \\ ${ }^{1}$ Department of Orthopaedic Surgery, Faculty of Medicine Siriraj Hospital, Mahidol University, Bangkok; \\ ${ }^{2}$ Department of Orthopaedics, Faculty of Medicine, Naresuan University, Phitsanulok, Thailand
}

Corresponding author

Aasis Unnanuntana

Department of Orthopaedic Surgery, Faculty

of Medicine Siriraj Hospital, Mahidol

University, 2 Wang Lang Road, Bangkok Noi,

Bangkok 10700, Thailand

Tel: +66-2-419-7968

Fax: +66-2-419-7967

E-mail: uaasis@gmail.com

Received: March 17, 2021

Revised: April 10, 2021

Accepted: April 19, 2021
Paget's disease of bone (PDB) is a progressive bone disorder characterized by increased osteoclast-mediated bone resorption and abnormal bone formation. Incomplete atypical femoral fracture, appearing radiographically as a stress fracture at the lateral aspect of the femur, is an uncommon low-trauma fracture frequently seen in association with long-term bisphosphonate therapy. We describe the case of a 61-year-old female patient with PDB who developed a stress fracture at the lateral femoral cortex after 5 doses of intravenous bisphosphonate. The conservative treatment plan included discontinuation of bisphosphonate, a continuation of calcium and vitamin D supplementation, and limited weight-bearing for 3 months. The patient's pain level gradually improved after switching to the new treatment plan. At the latest follow-up, approximately 5 years after the initiation of conservative treatment, the patient remained pain-free, and her PDB was well-controlled. However, the fracture line was still visible on the most recent radiograph. Although it remains unclear whether a stress fracture at the lateral femoral cortex occurred due to bisphosphonate therapy or PDB, this case highlights the importance of careful evaluation of any lesion that appears in PDB patients receiving bisphosphonate therapy.

Key Words: Femur · Fracture healing · Fractures, stress · Osteitis deformans · Paget's disease of bone

\section{INTRODUCTION}

The effectiveness of bisphosphonate has made it a mainstream drug for the treatment of osteoporosis. Additional indications for prescribing bisphosphonate include hypercalcemia of malignancy, multiple myeloma, bony metastasis, and Paget's disease of bone (PDB).[1] PDB is a chronic bone disorder characterized by increased bone resorption and abnormal bone formation. PDB mainly effects axial skeleton including pelvis, femur, lumbar spine, skull, and tibia. The condition is uncommon in Asia and affects middle-aged or older adults.[2,3]

The indication of bisphosphonate in PDB is to control symptoms. These encompass pain at the lesion site, symptoms due to cord or nerve compression, hearing loss, and substantial skeletal involvement causing heart failure.[4] Literatures, however, showed potential complications of stress fracture associated with its prolonged bisphosphonate use.[5] Atypical femoral fracture (AFF) is a rare type of stress fracture and is often associated with long-term bisphosphonate therapy. 
Stress fracture of the femoral shaft is uncommon and could occur at any sites along the femoral shaft between the subtrochanteric and supracondylar areas and involve lateral femoral cortex at initial presentation. Therefore, careful risk and benefit should be balanced while determining the appropriate candidate for using bisphosphonate therapy.

\section{CASE REPORT}

A 61-year-old Thai woman presented with a 1-year history of progressive pain, warm sensation, and enlargement with anterolateral bowing deformity of right leg, which led to walking difficulty that required a walker for ambulation. Plain radiographs of her right leg demonstrated anterolateral bowing of the right tibia with generalized osteolytic and osteoblastic lesions of the entire tibia (Fig. 1). Biochemical bone turnover markers showed an increased serum alkaline phosphatase level of $739 \mathrm{U} / \mathrm{L}$, increased serum Cterminal telopeptide of type I collagen (CTX) level of 1.310 $\mathrm{ng} / \mathrm{mL}$, and increased total procollagen type $1 \mathrm{~N}$-terminal propeptide (PINP) level of $>1,200 \mathrm{ng} / \mathrm{mL}$. Biopsy of the right tibia was performed, which revealed accelerated deposition of lamellar bone in a disorganized fashion (jigsaw puzzle-like or mosaic pattern) (Fig. 2). The clinical presen-
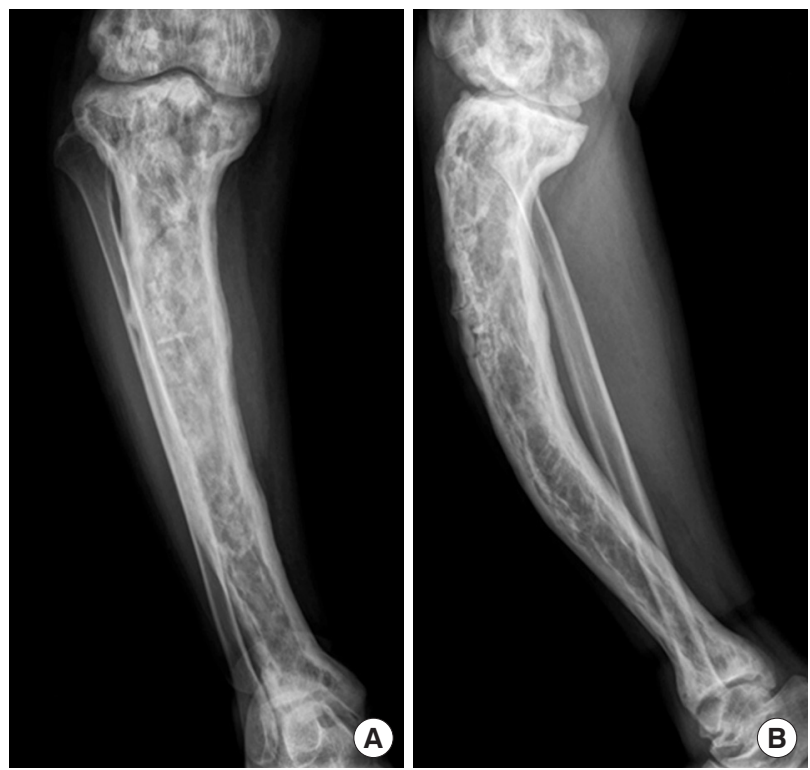

Fig. 1. Anteroposterior (A) and lateral $(B)$ radiographs of right leg showing anterolateral bowing of the tibia with classic radiographic features of Paget's disease of bone. Those features include generalized osteolytic and osteoblastic lesion, coarsening of trabeculae, cortical thickening, and diffuse bony expansion. tation, radiographic features, and laboratory tests led to a diagnosis of PDB. The patient was then treated with intravenous zoledronate $5 \mathrm{mg}$. After the initial administration of intravenous bisphosphonate, her clinical condition was dramatically improved and her pain was significantly decreased from a pain-visual analogue scale of 7 to 1 . Followup laboratory tests showed a significant reduction in all bone turnover markers. However, her pain subsided for only a short period of time. After 4 weeks of intravenous zoledronate administration, her pain and warm sensation returned to the same levels of intensity that she was experiencing before the initiation of bisphosphonate. Intravenous zoledronate was then readministered to control her symptoms. The symptoms subsided each time bisphosphonate was given.

After the administration of 5 doses of intravenous bisphosphonate within a 6-month period, she developed a new type of pain over her left mid-thigh. The pain was mechanical and was relieved with rest but was exacerbated while standing and walking. She denied any history of trauma prior to onset of this new mid-thigh pain. She was then referred to the metabolic bone disease (MBD) clinic at our orthopaedic center. Plain radiographs of her left femur was sent and revealed an incomplete transverse radiolucent line at the lateral cortex of the femoral shaft, which was compatible with a stress fracture (Fig. 3A). Magnetic reso-

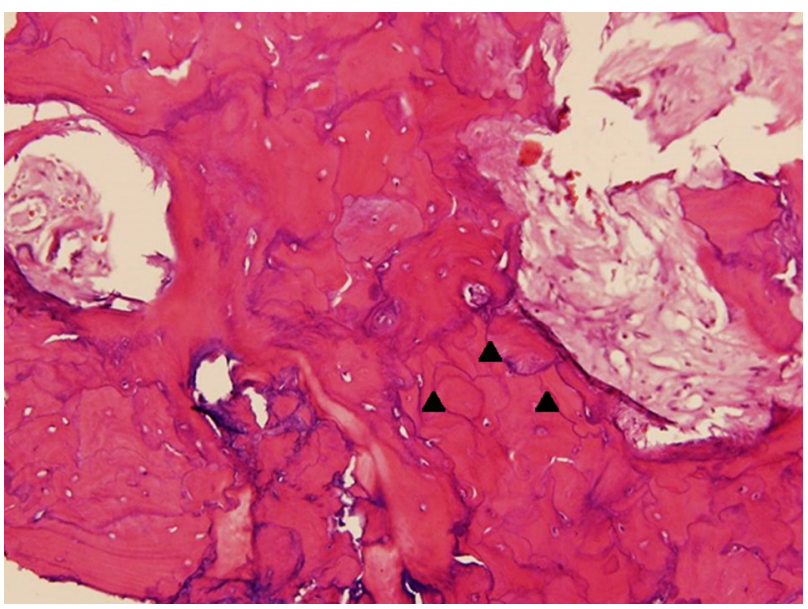

Fig. 2. Hematoxylin and eosin (H\&E) staining of bone tissue (magnification, $\times 100)$ from right tibia showing multiple cement lines (small black arrowheads) in marrow fibrosis that resemble a jigsaw puzzlelike or mosaic pattern (photograph courtesy of Soranart Muangsomboon, MD [Department of Pathology, Faculty of Medicine Siriraj Hospital, Mahidol University, Bangkok, Thailand]) 
nance imaging (MRI) was performed, which confirmed the presence of a stress fracture at midshaft of the left femur (Fig. 3B).

At the MBD clinic, intravenous zoledronate was stopped and conservative treatment with non-weight ambulation was advised. Calcium and vitamin D supplementation was continued. During the 6-week period after discontinuation of bisphosphonate, her pain had gradually subsided. Her pain continued to improve, so progressive weight-bearing from partial- to full-weight bearing with a walker was started at 3 months. At the most recent follow-up of 5 years after the discontinuation of bisphosphonate treatment, the patient remains pain-free even though the follow-up radiograph still shows a fracture line at the lateral femoral

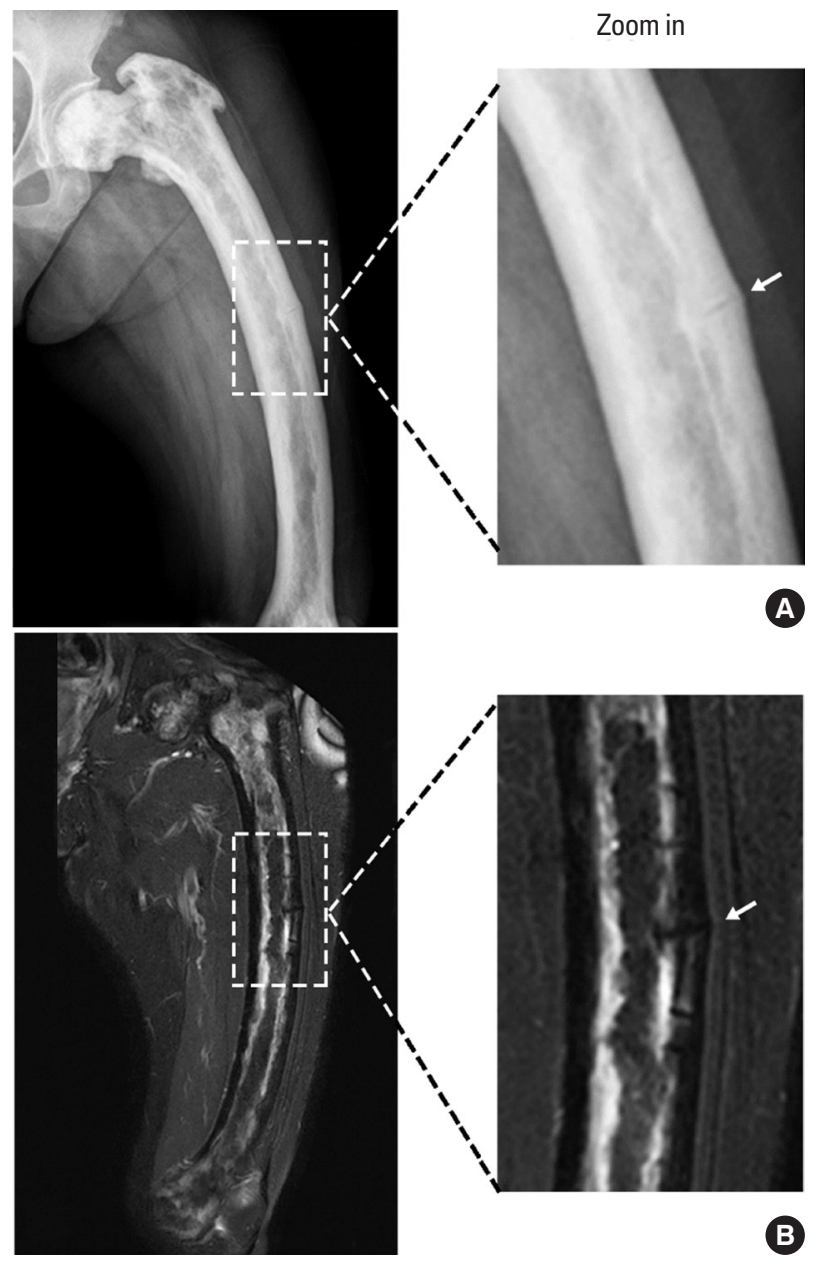

Fig. 3. Plain radiograph of left femur revealed an incomplete fracture line (white arrow) at the lateral femoral cortex (A). T1-weighted image with gadolinium contrast magnetic resonance imaging of the left femur confirms the presence of the fracture line at the lateral femoral cortex (B). cortex. She is able to ambulate independently with only the occasional use of a cane. She has no symptoms of stress fracture and her PDB is well controlled. The latest laboratory tests, including serum alkaline phosphatase, serum CTX, and PINP, are within normal limits (Fig. 4).

\section{DISCUSSION}

Incomplete AFF or stress fracture at the lateral femoral cortex falls into a rare category of fracture, the incidence of which is not well established.[6,7] The revised case definition of AFF was proposed by the American Society for Bone and Mineral Research (ASBMR) task force 2013. At least 4 out of 5 major features must be present, while none of the minor features is required for a diagnosis of complete AFF. [8] Pathogenic causes, such as biomechanical, material, and microdamage, are evident, and these contribute to the development and consequences of AFF. Different factors, such as metabolic bone disorders (e.g., X-linked hypophosphatemia, rickets and osteopetrosis) and steroids, can contribute to the development of stress fracture in some patients.[9-13] Genetic factors can influence bone geometry, as evidenced by the changes to the femur in our patient that increased its curvature. This leads to an increase in tensile load, which increases the risk of stress fracture at the lateral femoral cortex.[14] Additionally, altered lower limb alignment causes continuous mechanical loading (e.g., coxa vara), which has been associated with a stress fracture.[15,16] Furthermore, the site of the stress fracture has been shown to be affected by the anterolateral bowing of the femur; the definite explanation is however unclear.[17] Additionally, although the revised definition of AFF by the ASBMR excludes MBDs (e.g., PDB), a stress fracture can occur in PDB patients who received long-term bisphosphonate treatment. This is due to the reason that bisphosphonate may increase the risk of stress fracture by decreasing bone turnover. This causes an accumulation of bone at sites of high osteogenic activity, which causes alteration of bone mineralization and loss of bone heterogeneity, which ultimately leads to a stress fracture. $[9,18]$

$\mathrm{PDB}$ is a progressive bone disorder where the interactions between both environmental and genetic factors have been identified as important factors for its development.[19] PDB is characterized by impaired bone metabolism due to excessive bone remodeling. In PDB, excessive over-activity of 


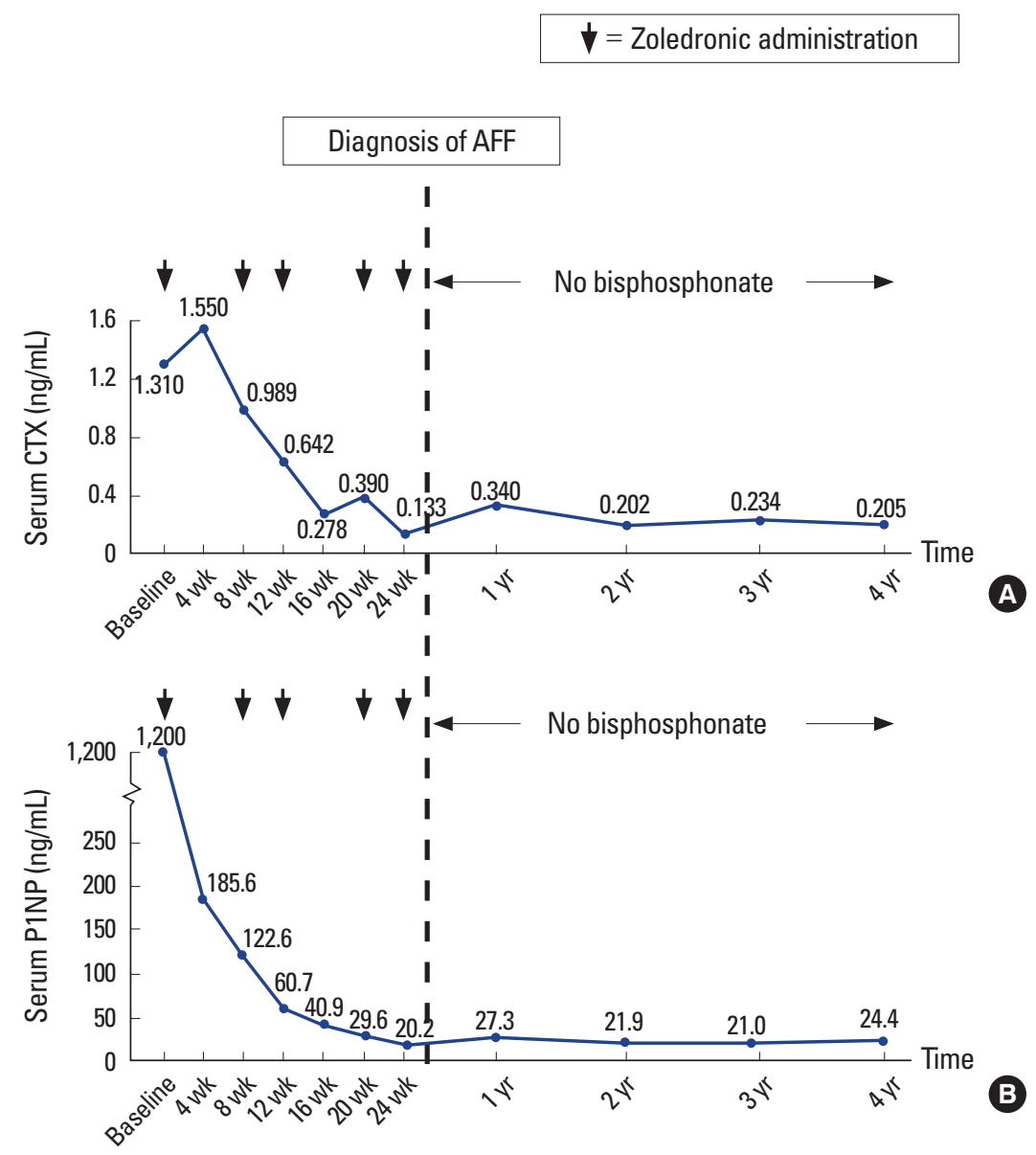

Fig. 4. Graphs showing biochemical bone turnover marker responses of serum C-terminal telopeptide of type I collagen (CTX) (A) and procollagen type $1 \mathrm{~N}$-terminal propeptide (PINP) (B) after the patient received 5 doses of intravenous bisphosphonate. The vertical dotted line indicates when the patient developed stress fracture at the lateral femoral cortex. Bisphosphonate was then discontinued, after which both serum CTX and serum P1NP remained stable within a premenopausal range throughout 4-year follow-up period.

osteoblasts and osteoclasts might also result in the deposition of low-quality woven bone or area of osteolysis, resulting in deformity, secondary osteoarthritis and fracture. [20] It is presumed that stress fracture might occur in patients with PDB.[20,21] Although the exact etiology remains unclear, there are few possible explanations. First, bisphosphonate is a strong precipitating factor that is associated with a stress fracture. Odvina et al. [22] proposed that bisphosphonate-associated stress fracture occurs as a result of over suppression of bone turnover, which leads to microdamage accumulation that causes a stress fracture. Second, a disease process that results in femoral bowing causes an increase in stress concentration that can lead to insufficiency fracture in the high tensile load area.[23] Accordingly, we couldn't and are still unable to determine whether the stress fracture occurred as a result of PDB or the 5 doses of zoledronate. However, consideration of all of the evaluable factors leads us to believe that the cause was multifactorial. This is because the patient had significant lateral femoral bowing that caused stress concentration at the lateral femoral cortex. This excessive femoral bowing increased the bending force, which likely led to the development of stress fracture. In addition, our patient received multiple doses of bisphosphonate. Thus, we presume that bisphosphonate might inhibit the healing process of stress fracture at the lateral femoral cortex.

In this case, the patient received 5 doses of zoledronate within a 6-month time period. Since similar symptoms reappeared, repeated administration of the drug was performed to control the patient's symptoms. The drug was administered at this frequency because the patient's symptoms improved for approximately only 1 month after each 
bisphosphonate administration. It is important to point out that bisphosphonate administration in this patient did not follow an Endocrine Society Clinical Practice Guideline 2014 for the treatment of PDB, which suggests a single 5-mg dose of intravenous zoledronate as the treatment of choice for PDB patients and retreatment is seldom required within 5 years after the initial dose of intravenous zoledronate. [24] The previous article has also shown the effectiveness of treatment in PDB by the use of intravenous zoledronate. [25] The reason for such initial management in this patient is due to the reason that PDB is fairly uncommon in Asian countries especially in Thailand; therefore, the clinician might not have been aware of the currently available guidelines and has little experience of treating PDB patients. Nevertheless, the objective of this case report is to show the management of stress fracture in PDB rather than a treatment that was done before being sent to our MBD clinic.

Generally, bisphosphonate is an effective treatment for PDB, allowing normalization of bone turnover markers and improvement in quality of life.[26] If pain persists or recurs after bisphosphonate therapy, further imaging studies e.g. repeated radiographs or MRI should be performed to rule out the following conditions: neoplastic change, degenerative joint disorder or the occurrence of a stress fracture as shown in this case report.[27-29]

There is limited evidence in the literature describing stress fracture in PDB. A 78-year-old female with PDB who received alendronate for 16 years and developed stress fracture following low-energy trauma was previously reported.[21] That present case report highlights questions relating to whether bone-in PDB is at risk due to decreased tensile strength and whether stress fracture is caused by bisphosphonate or by the natural course of PDB.

Regarding treatment for a stress fracture, in general, bisphosphonate should be stopped in patients who develop a bisphosphonate-associated stress fracture, and teriparatide should be administered. However, we could not give teriparatide to this patient because she has PDB and teriparatide is contraindicated in patients with this condition. For surgical treatment of stress fracture, fixation with an intramedullary nail is usually indicated for a complete fracture, or as a prophylactic treatment in painful stress fracture.[30] Regarding the type of nail, a full-length intramedullary nail is preferred [31]; however, a partial-length intramedullary nail has also shown similar outcomes as full- length nail.[32] In this case, we chose to treat our patient conservatively due to her severe lateral femoral bowing. More specifically, insertion of a relatively straight intramedullary nail into a curved bone would be technically challenging and may not effectuate the intended effect. Conservative treatment in this case included discontinuation of bisphosphonate, continuation of calcium and vitamin D supplementation, and limited weight bearing for 3 months. Her symptoms gradually improved over the first 6 weeks of conservative treatment. At the latest follow-up of 5 years after the discontinuation of bisphosphonate, the patient is pain-free and can ambulate independently with only occasional use of a cane although the fracture line is still visible from the most recent follow-up radiograph. Similar to our study, Png et al. [33] reported that a stress fracture at the lateral femoral cortex could persist even after discontinuation of bisphosphonate.

Once stress fracture occurs in a patient with PDB, treatment to control PDB is difficult. In general, bisphosphonate is the first-line medication for the treatment of PDB. Treatment of PDB was challenging in our patient because she had already developed a stress fracture at the lateral femoral cortex. We determined continued bisphosphonate treatment to be inappropriate since her stress fracture developed after the administration of intravenous bisphosphonate. Therefore, calcitonin may be an alternative treatment option in patients with PDB who have already developed a stress fractures. Although calcitonin is the first effective medication used for the treatment of PDB, the requirement of daily injection and the frequency occurrence of flushing and nausea limited its acceptability to use in PDB patients. [24] Nevertheless, calcitonin could be considered for shortterm treatment of bone pain in PDB when bisphosphonate is contraindicated.[34] A newer therapeutic regime that acts at the genetic level may be a promising future treatment prospect. Studies have already shown that a vast number of genes are regulated in PDB.[35] The development of drugs to target specific genes may improve patient treatment outcomes by improving patient compliance with minimal or no risk.

The limitations of this study must be described. First, we were unable to conclusively establish whether a stress fracture that developed in our patient was caused by a combination of severe lateral femoral bowing and bisphosphonate or as a result of the natural course of PDB. Since there 
is persistent visibility of the fracture line at the lateral femoral cortex even after 5 years of discontinuation of bisphosphonate, it is possible that stress fracture occurred as a result of the disease process of PDB. Second, we were unable to explain the reason why the typical symptom of stress fracture occurred after only 5 doses of bisphosphonate therapy. Third, comparison of the femoral shaft before and after administration of bisphosphonate was not possible due to a scarcity of baseline radiographs and the fact that the baseline radiographic images were of the proximal part of the left femur only. Moreover and importantly, since the lesion is inconspicuous radiographically, there is a possibility that the fracture line was missed earlier and that it was present before bisphosphonate administration.

In conclusion, we describe a patient who was diagnosed with PDB that received intravenous bisphosphonate therapy but later developed a stress fracture. Although the cause of stress fracture in this patient is still unclear, this case highlights the importance of careful monitoring of patients with PDB for a stress fracture, especially in patients having lateral femoral bowing deformity that may lead to stress accumulation in that area and in those who receive prolonged or high-dose bisphosphonate. If a stress fracture occurred in patient with PDB, conservative management including a period of protected weight-bearing, rest, calcium and vitamin D supplementation, and stoppage of bisphosphonate use can be successfully performed.

\section{DECLARATIONS}

\section{Acknowledgments}

The authors gratefully acknowledge the patient profiled in this report for granting us permission to disclose details relating to her case, and Ms. Wachirapan Narktang for her assistance with figure development.

\section{Funding}

The authors received no financial support for this article.

\section{Ethics approval and consent to participate Not applicable.}

\section{Conflict of interest}

No potential conflict of interest relevant to this article was reported.

\section{ORCID}

Sarthak Nepal https://orcid.org/0000-0003-2039-1443

Atthakorn Jarusriwanna

https://orcid.org/0000-0002-3919-8195

Aasis Unnanuntana https://orcid.org/0000-0002-5742-298X

\section{REFERENCES}

1. Drake MT, Clarke BL, Khosla S. Bisphosphonates: mechanism of action and role in clinical practice. Mayo Clin Proc 2008;83:1032-45. https://doi.org/10.4065/83.9.1032.

2. Takigami I, Ohara A, Matsumoto K, et al. Functional bracing for delayed union of a femur fracture associated with Paget's disease of the bone in an Asian patient: a case report. J Orthop Surg Res 2010;5:33. https://doi.org/10.1186/ 1749-799x-5-33.

3. Nebot Valenzuela E, Pietschmann P. Epidemiology and pathology of Paget's disease of bone - a review. Wien Med Wochenschr 2017;167:2-8. https://doi.org/10.1007/s10354016-0496-4.

4. Wat WZ. Current perspectives on bisphosphonate treatment in Paget's disease of bone. Ther Clin Risk Manag 2014; 10:977-83. https://doi.org/10.2147/tcrm.S58367.

5. Reddy SV. Long term bisphosphonate therapy \& atypical femoral fractures: a word of caution. Indian J Med Res 2014; 140:8-10.

6. Toro G, Ojeda-Thies C, Calabrò G, et al. Management of atypical femoral fracture: a scoping review and comprehensive algorithm. BMC Musculoskelet Disord 2016;17:227. https://doi.org/10.1186/s12891-016-1086-8.

7. Lloyd AA, Gludovatz B, Riedel C, et al. Atypical fracture with long-term bisphosphonate therapy is associated with altered cortical composition and reduced fracture resistance. Proc Natl Acad Sci U S A 2017;114:8722-7. https://doi.org/ 10.1073/pnas.1704460114.

8. Shane E, Burr D, Abrahamsen B, et al. Atypical subtrochanteric and diaphyseal femoral fractures: second report of a task force of the American Society for Bone and Mineral Research. J Bone Miner Res 2014;29:1-23. https://doi.org/ 10.1002/jbmr.1998.

9. McKenna MJ, Heffernan E, Hurson C, et al. Clinician appro ach to diagnosis of stress fractures including bisphosphonate-associated fractures. QJM 2014;107:99-105. https:// doi.org/10.1093/qjmed/hct192.

10. Meléndez LM, Llorente I, García E, et al. SAT0288 atypical 
femoral fractures after treatment with bisphosphonates and denosumab in inflammatory and senile osteoporosis. Ann Rheum Dis 2015;74:762-3. https://doi.org/10.1136/ annrheumdis-2015-eular.5000.

11. Marini F, Brandi ML. Atypical femur fractures: a distinctive tract of adult hypophosphatasia. Clin Cases Miner Bone Metab 2017;14:324-8. https://doi.org/10.11138/ccmbm/ 2017.14.3.324.

12. Larsen MS, Schmal H. The enigma of atypical femoral fractures: A summary of current knowledge. EFORT Open Rev 2018;3:494-500. https://doi.org/10.1302/2058-5241.3.170070.

13. Nguyen $\mathrm{HH}$, van de Laarschot $\mathrm{DM}$, Verkerk $A$, et al. Genetic risk factors for atypical femoral fractures (AFFs): A systematic review. JBMR Plus 2018;2:1-11. https://doi.org/10.1002/ jbm4.10024.

14. Saita Y, Ishijima M, Kaneko K. Atypical femoral fractures and bisphosphonate use: current evidence and clinical implications. Ther Adv Chronic Dis 2015;6:185-93. https:// doi.org/10.1177/2040622315584114.

15. Hagen JE, Miller AN, Ott SM, et al. Association of atypical femoral fractures with bisphosphonate use by patients with varus hip geometry. J Bone Joint Surg Am 2014;96: 1905-9. https://doi.org/10.2106/jbjs.N.00075.

16. Taormina DP, Marcano Al, Karia R, et al. Symptomatic atypical femoral fractures are related to underlying hip geometry. Bone 2014;63:1-6. https://doi.org/10.1016/j.bone.2014. 02.006 .

17. Park YC, Yoon SP, Yang KH. Localization of atypical femoral fracture on straight and bowed femurs. J Bone Metab 2019; 26:123-31. https://doi.org/10.11005/jbm.2019.26.2.123.

18. Ettinger B, Burr DB, Ritchie RO. Proposed pathogenesis for atypical femoral fractures: lessons from materials research. Bone 2013;55:495-500. https://doi.org/10.1016/j.bone.2013. 02.004 .

19. Galson DL, Roodman GD. Pathobiology of Paget's disease of bone. J Bone Metab 2014;21:85-98. https://doi.org/10. 11005/jbm.2014.21.2.85.

20. Kang H, Park YC, Yang KH. Paget's disease: Skeletal manifestations and effect of bisphosphonates. J Bone Metab 2017;24:97-103. https://doi.org/10.11005/jbm.2017.24. 2.97.

21. Kilcoyne A, Heffernan EJ. Atypical proximal femoral fractures in patients with paget disease receiving bisphosphonate therapy. AJR Am J Roentgenol 2011;197:W196-7. https: //doi.org/10.2214/ajr.10.6343.
22. Odvina CV, Zerwekh JE, Rao DS, et al. Severely suppressed bone turnover: a potential complication of alendronate therapy. J Clin Endocrinol Metab 2005;90:1294-301. https: //doi.org/10.1210/jc.2004-0952.

23. Yoo H, Cho Y, Park Y, et al. Lateral femoral bowing and the location of atypical femoral fractures. Hip Pelvis 2017;29: 127-32. https://doi.org/10.5371/hp.2017.29.2.127.

24. Singer FR, Bone HG, 3rd, Hosking DJ, et al. Paget's disease of bone: an endocrine society clinical practice guideline. J Clin Endocrinol Metab 2014;99:4408-22. https://doi.org/ 10.1210/jc.2014-2910.

25. Kim KC. Intravenous zoledronate for a patient with Paget's disease. J Bone Metab 2014;21:223-6. https://doi.org/10. 11005/jbm.2014.21.3.223.

26. Reid IR, Miller P, Lyles K, et al. Comparison of a single infusion of zoledronic acid with risedronate for Paget's disease. N Engl J Med 2005;353:898-908. https://doi.org/10.1056/ NEJMoa044241.

27. Winfield J, Stamp TC. Bone and joint symptoms in Paget's disease. Ann Rheum Dis 1984;43:769-73. https://doi.org/ 10.1136/ard.43.6.769.

28. López C, Thomas DV, Davies AM. Neoplastic transformation and tumour-like lesions in Paget's disease of bone: a pictorial review. Eur Radiol 2003;13 Suppl 4:L151-63. https: //doi.org/10.1007/s00330-003-1927-3.

29. Matiotti SB, Tramunt CS, Duarte RD, et al. Sarcomatous degeneration of Paget's disease in the calcaneus: A case report. Radiol Bras 2009;42:63-5. https://doi.org/10.1590/ S0100-39842009000100013.

30. Park YC, Song HK, Zheng XL, et al. Intramedullary nailing for atypical femoral fracture with excessive anterolateral bowing. J Bone Joint Surg Am 2017;99:726-35. https:// doi.org/10.2106/jbjs.16.00760.

31. Shane E, Burr D, Ebeling PR, et al. Atypical subtrochanteric and diaphyseal femoral fractures: report of a task force of the American Society for Bone and Mineral Research. J Bone Miner Res 2010;25:2267-94. https://doi.org/10.1002/ jbmr.253.

32. Kim KK, Ryu SK, Lee SW, et al. Is full-length intramedullary nail necessary for atypical subtrochanteric femoral fracture associated with bisphosphonate? J Bone Metab 2020;27: 133-42. https://doi.org/10.11005/jbm.2020.27.2.133.

33. Png MA, Mohan PC, Koh JSB, et al. Natural history of incomplete atypical femoral fractures in patients after a prolonged and variable course of bisphosphonate therapy-a 
long-term radiological follow-up. Osteoporos Int 2019;30: 2417-28. https://doi.org/10.1007/s00198-019-05067-7.

34. Ralston SH, Corral-Gudino L, Cooper C, et al. Diagnosis and management of Paget's disease of bone in adults: A clinical guideline. J Bone Miner Res 2019;34:579-604. https:// doi.org/10.1002/jbmr.3657.

35. Michou L, Brown JP. Emerging strategies and therapies for treatment of Paget's disease of bone. Drug Des Devel Ther 2011;5:225-39. https://doi.org/10.2147/dddt.S11306. 